Supporting Information

\title{
Synthesis and Chiroptical Properties of Planar Chiral Azahelicenes Based on [2.2]Paracyclophane
}

Dan $\mathrm{Xu}$, Wen-Hua Zheng*

State Key Laboratory of Coordination Chemistry, Jiangsu Key Laboratory of Advanced Organic Materials, School of Chemistry and Chemical Engineering, Nanjing University, 163 Xianlin Avenue,

Nanjing 210023, Jiangsu, China

Table of Contents

General Information................................................S2

Synthetic Procedures and Characterization Data................S2-S6

References.........................................................S7

Theoretical Calculations........................................S8-S11

X-ray Crystallographic Data for 3c.................................S11-S12

NMR and HRMS Spectra..................................S13-S24 


\section{General Information}

Unless stated otherwise, all reactions were carried out in flame-dried glassware under a dry nitrogen atmosphere. All solvents were purified and dried according to standard methods prior to use. Melting points were measured on a SGW X-4. Melting points are uncorrected. NMR spectra were recorded on a Bruker ARX 500 spectrometer and were recorded in ppm $(\delta)$ downfield of TMS $(\delta=0)$ in deuterated solvent. Signal splitting patterns are described as singlet (s), doublet (d), triplet (t), quartet (q), quintet (quint), or multiplet (m), with coupling constants (J) in hertz. Mass spectra were conducted at Micromass Q-Tof instrument (ESI) and Agilent Technologies 5973N (EI). Values of optical rotation were measured on Rudolph Automatic Polarimeter A21101 at the wavelength of the sodium D-line $(589 \mathrm{~nm})$. UV spectra were obtained on a UV-3100. Fluorescence spectra were recorded on a Hitachi F-4600 spectrophotometer. Circular dichroism spectra were measured with JASCO J-810, circular polarized luminance spectra were obtained with JASCO CPL-300.

\section{Synthetic Procedures and Characterization data}

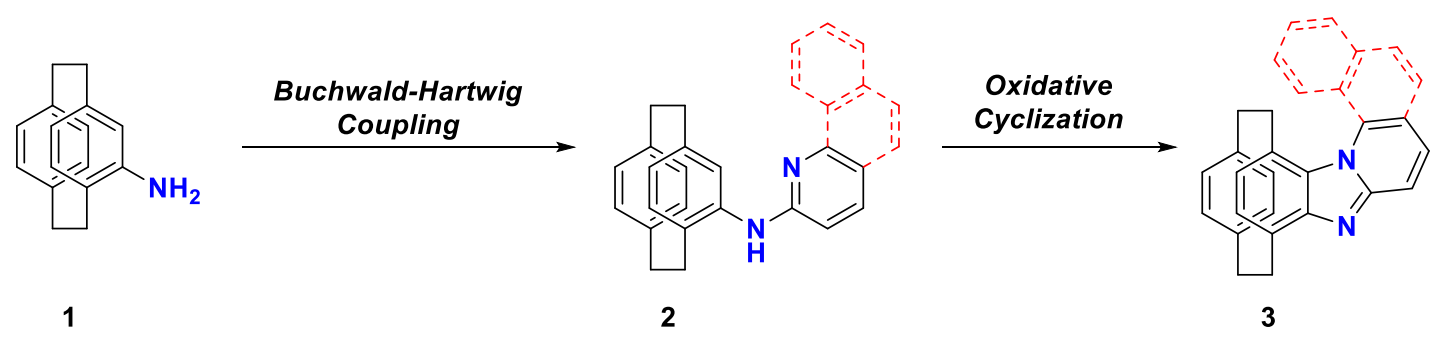

\section{General Procedure for Preparation of $2 \mathbf{a}^{-1}{ }^{1}$ :}

To a $10 \mathrm{~mL}$ Schlenk tube were added chiral 4-amino[2.2]paracyclophane $\left(\boldsymbol{R}_{\boldsymbol{p}}\right) /\left(\boldsymbol{S}_{\boldsymbol{p}}\right) \mathbf{- 1}$ (100.5 mg, $0.45 \mathrm{mmol}, 1.0$ equiv), 2-halogenated pyridine derivatives $(0.47 \mathrm{mmol}$, 1.05 equiv)), $\mathrm{Cs}_{2} \mathrm{CO}_{3}$ (439.8 mg, $1.35 \mathrm{mmol}, 3.0$ equiv), $\mathrm{Pd}(\mathrm{OAc})_{2}$ (4.0 mg, 0.018 mmol, $4 \mathrm{~mol} \%$ ), rac-BINAP (11.2 $\mathrm{mg}, 0.018 \mathrm{mmol}, 4 \mathrm{~mol} \%$ ) and anhydrous toluene $(5 \mathrm{~mL})$, and the resulting mixture was stirred under a nitrogen atmosphere at $100{ }^{\circ} \mathrm{C}$ in an oil bath overnight. The solution was transferred to a separatory funnel and washed with saturated aqueous ammonium chloride and water. The organic layer was separated, dried over $\mathrm{Na}_{2} \mathrm{SO}_{4}$ and concentrated. The resulting residue was purified by flash column chromatography (petroleum ether /ethyl acetate) to afford on silica gel to give the product $\mathbf{2} \mathbf{a}-\mathbf{c}$.

\section{General Procedure for Preparation of $3 \mathbf{a} / 3 \mathbf{b}^{2}$ :}

A solution of $\mathbf{2 a} / \mathbf{2 b}(0.1 \mathrm{mmol}, 1.0$ equiv) and Selectfluor $(53.1 \mathrm{mg}, 0.15 \mathrm{mmol}, 1.5$ equiv) in HFIP $(2 \mathrm{~mL})$ and $\mathrm{H}_{2} \mathrm{O}(1 \mathrm{~mL})$ was stirred at room temperature for $5 \mathrm{~min}$, 1-iodo-4-methoxybenzene $(35.1 \mathrm{mg}, 0.15 \mathrm{mmol}, 1.5$ equiv) was added and the reaction mixture was stirred at room temperature for $2 \mathrm{~h}$. The solution was quenched with saturated sodium bicarbonate solution $(15 \mathrm{~mL})$, extracted with DCM, dried over 
$\mathrm{Na}_{2} \mathrm{SO}_{4}$ and concentrated under vacuum. The product $\mathbf{3 a} / \mathbf{b}$ was obtained after flash column chromatography (petroleum ether /ethyl acetate) as yellow solid.

\section{Procedure for Preparation of $3 c^{3}$ :}

A solution of $2 \mathbf{c}\left(40.1 \mathrm{mg}, 0.1 \mathrm{mmol}, 1.0\right.$ equiv) and $\mathrm{PhI}\left(\mathrm{OCOCF}_{3}\right)_{2}(55.9 \mathrm{mg}, 0.13$ mmol, 1.3 equiv) in HFIP ( $1 \mathrm{~mL})$ was stirred at room temperature for $3 \mathrm{~h}$. The solution was quenched with saturated sodium bicarbonate solution $(15 \mathrm{~mL})$, extracted with DCM, dried over $\mathrm{Na}_{2} \mathrm{SO}_{4}$ and concentrated under vacuum. The product $3 \mathbf{c}$ was obtained after flash column chromatography (petroleum ether /ethyl acetate) as a yellow solid.

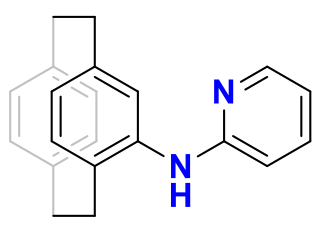

$\left(S_{p}\right)-2 a$

$\left(S_{p}\right)$-N-(1,4(1,4)-dibenzenacyclohexaphane-12-yl)pyridin-2-amine [( $\left.\left.S_{p}\right)-2 \mathrm{a}\right]$ :

Yellow solid, $105.0 \mathrm{mg}, 78 \%$ yield $(\mathrm{PE} / \mathrm{EA}=10 / 1)$. Analytical data for $\left(\boldsymbol{S}_{\boldsymbol{p}}\right)$-2a: $\mathrm{mp}$ : 145-147 ${ }^{\circ} \mathrm{C}$; ${ }^{1} \mathrm{H}$ NMR $\left(500 \mathrm{MHz}, \mathrm{CDCl}_{3}\right) \delta 8.19$ (ddd, $\left.J=5.1,1.8,0.8 \mathrm{~Hz}, 1 \mathrm{H}\right)$, 7.46-7.39 (m, 1H), $7.02(\mathrm{dd}, J=7.9,1.9 \mathrm{~Hz}, 1 \mathrm{H}), 6.75-6.69(\mathrm{~m}, 1 \mathrm{H}), 6.63(\mathrm{~d}, J=8.5$ $\mathrm{Hz}, 1 \mathrm{H}), 6.57$ (dd, $J=7.8,1.9 \mathrm{~Hz}, 1 \mathrm{H}), 6.51(\mathrm{~d}, J=7.8 \mathrm{~Hz}, 1 \mathrm{H}), 6.49-6.44(\mathrm{~m}, 3 \mathrm{H})$, $6.06(\mathrm{~d}, J=1.5 \mathrm{~Hz}, 1 \mathrm{H}), 3.13-2.93(\mathrm{~m}, 7 \mathrm{H}), 2.76-2.69(\mathrm{~m}, 1 \mathrm{H}),{ }^{13} \mathrm{C} \mathrm{NMR}(126 \mathrm{MHz}$, $\left.\mathrm{CDCl}_{3}\right) \delta 156.4,148.1,141.7,139.7,139.2,138.3,137.8,136.0,134.0,133.7,133.0$, 131.8, 129.5, 128.9, 128.1, 114.9, 107.4, 35.3, 35.0, 34.1, 33.8; IR (neat) 3210, 2924, 2851, 1592, 1434, 1322, 1151, 767, 716, $512 \mathrm{~cm}^{-1}$; HRMS (ESI) m/z: calcd for $\mathrm{C}_{21} \mathrm{H}_{19} \mathrm{~N}_{2}[\mathrm{M}-\mathrm{H}]^{-}, 299.1554$; found, 299.1542. $[\alpha]_{\mathrm{D}}{ }^{20}=R_{p} / S_{p}-41.6 /+45.0$ (c 0.49 , $\left.\mathrm{CHCl}_{3}\right)$.<smiles>c1ccc2c(c1)CCC2</smiles>

$\left(S_{p}\right)-2 b$

$\left(S_{p}\right)$-N-(1,4(1,4)-dibenzenacyclohexaphane-12-yl)quinolin-2-amine [ $\left(S_{p}\right)$-2b]:

Yellow solid, $131.2 \mathrm{mg}, 83 \%$ yield $(\mathrm{PE} / \mathrm{EA}=10 / 1)$. Analytical data for $\left(\boldsymbol{S}_{\boldsymbol{p}}\right)-\mathbf{2 b}: \mathrm{mp}$ : 109-111 ${ }^{\circ} \mathrm{C} ;{ }^{1} \mathrm{H}$ NMR $\left(500 \mathrm{MHz}, \mathrm{CDCl}_{3}\right) \delta 7.85(\mathrm{~d}, J=8.9 \mathrm{~Hz}, 1 \mathrm{H}), 7.77(\mathrm{~d}, J=8.4$ $\mathrm{Hz}, 1 \mathrm{H}), 7.66-7.58(\mathrm{~m}, 2 \mathrm{H}), 7.32-7.26(\mathrm{~m}, 1 \mathrm{H}), 7.06(\mathrm{dd}, J=7.9,1.8 \mathrm{~Hz}, 1 \mathrm{H}), 6.87(\mathrm{~d}$, $J=9.0 \mathrm{~Hz}, 1 \mathrm{H}), 6.80(\mathrm{~s}, 1 \mathrm{H}), 6.60(\mathrm{dd}, J=7.8,1.8 \mathrm{~Hz}, 1 \mathrm{H}), 6.56(\mathrm{~d}, J=7.8 \mathrm{~Hz}, 1 \mathrm{H})$, $6.51(\mathrm{dd}, J=7.8,1.7 \mathrm{~Hz}, 1 \mathrm{H}), 6.48(\mathrm{dd}, J=7.9,1.9 \mathrm{~Hz}, 1 \mathrm{H}), 6.46(\mathrm{~d}, J=7.9 \mathrm{~Hz}, 1 \mathrm{H})$, $6.16(\mathrm{~d}, J=1.6 \mathrm{~Hz}, 1 \mathrm{H}), 3.18-2.94(\mathrm{~m}, 7 \mathrm{H}), 2.79-2.72(\mathrm{~m}, 1 \mathrm{H}) ;{ }^{13} \mathrm{C} \mathrm{NMR}(126 \mathrm{MHz}$, $\left.\mathrm{CDCl}_{3}\right) \delta 155.2,147.8,141.7,139.8,139.2,138.1,137.7,136.0,134.4,133.7,133.1$, 
$131.8,130.1,129.8,129.4,128.0,127.7,126.2,124.4,123.0,110.0,35.4,35.0,34.1$, 33.7; IR (neat) 3444, 2921, 2849, 1614, 1489, 1424, 1345, 1182, 1144, 826, 752, 532 $\mathrm{cm}^{-1}$; HRMS (ESI) m/z: calcd for $\mathrm{C}_{25} \mathrm{H}_{21} \mathrm{~N}_{2}$ [M-H] $]^{-}$349.1710; found, 349.1709. $[\alpha]_{\mathrm{D}}{ }^{20}=R_{p} / S_{p}-53.2 /+60.1\left(\mathrm{c} 1.0, \mathrm{CHCl}_{3}\right)$.<smiles>c1ccc2c(c1)CCC2</smiles>

$\left(S_{p}\right)-2 c$

$\left(S_{p}\right)$-N-(1,4(1,4)-dibenzenacyclohexaphane-12-yl)benzo[ $\left.h\right]$ quinolin-2-amine $\left[\left(S_{p}\right)-2 c\right]$ :

Yellow solid, $164.4 \mathrm{mg}, 91 \%$ yield $(\mathrm{PE} / \mathrm{EA}=10 / 1)$. Analytical data for $\left(\boldsymbol{S}_{\boldsymbol{p}}\right)-2 \mathrm{c}: \mathrm{mp}$ : 246-248 ${ }^{\circ} \mathrm{C} ;{ }^{1} \mathrm{H}$ NMR $\left(500 \mathrm{MHz}, \mathrm{CDCl}_{3}\right) \delta 9.26(\mathrm{~d}, J=8.1 \mathrm{~Hz}, 1 \mathrm{H}), 7.93(\mathrm{~d}, J=8.7$ $\mathrm{Hz}, 1 \mathrm{H}), 7.89(\mathrm{~d}, J=7.8 \mathrm{~Hz}, 1 \mathrm{H}), 7.72(\mathrm{t}, J=7.3 \mathrm{~Hz}, 1 \mathrm{H}), 7.67(\mathrm{t}, J=7.1 \mathrm{~Hz}, 1 \mathrm{H})$, $7.59(\mathrm{q}, J=7.6 \mathrm{~Hz}, 2 \mathrm{H}), 7.00(\mathrm{~d}, J=7.5 \mathrm{~Hz}, 1 \mathrm{H}), 6.95(\mathrm{~d}, J=8.7 \mathrm{~Hz}, 1 \mathrm{H}), 6.84(\mathrm{~s}$, $1 \mathrm{H}), 6.61(\mathrm{dd}, J=7.8,1.6 \mathrm{~Hz}, 1 \mathrm{H}), 6.56(\mathrm{~d}, J=7.8 \mathrm{~Hz}, 1 \mathrm{H}), 6.49(\mathrm{dd}, 2 \mathrm{H}), 6.42(\mathrm{~d}, J$ $=7.7 \mathrm{~Hz}, 1 \mathrm{H}), 6.36(\mathrm{~s}, 1 \mathrm{H}), 3.26-3.19(\mathrm{~m}, 1 \mathrm{H}), 3.11-3.04(\mathrm{~m}, 5 \mathrm{H}), 3.02-2.96(\mathrm{~m}, 1 \mathrm{H})$, 2.85-2.75 (m, 1H); ${ }^{13} \mathrm{C}$ NMR $\left(126 \mathrm{MHz}, \mathrm{CDCl}_{3}\right) \delta 154.7,146.0,141.5,139.7,139.2$, $138.3,137.9,135.9,134.4,133.7,133.5,133.0,131.8,130.7,129.1,128.3,128.1$, $127.9,126.3,125.4,124.6,123.6,121.1,109.2,35.4,35.1,34.2,33.9$; IR (neat) 3236, 2922, 2849, 1597, 1494, 1443, 1347, 1259, 1091, 1017, 798, $756 \mathrm{~cm}^{-1}$; HRMS (ESI) $\mathrm{m} / \mathrm{z}$ : calcd for $\mathrm{C}_{29} \mathrm{H}_{23} \mathrm{~N}_{2}[\mathrm{M}-\mathrm{H}]^{-}, 399.1867$; found, 399.1869. $[\alpha]_{\mathrm{D}}{ }^{20}=R_{p} / S_{p^{-}} 31.9 /+31.6$ (c $\left.0.68, \mathrm{CHCl}_{3}\right)$.

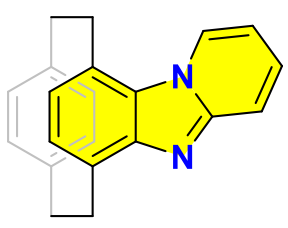

$$
\left(S_{p}\right)-3 a
$$

\section{$\left(S_{p}\right)$-1(6,9)-benzo[4,5]imidazo[1,2-a]pyridina-4(1,4)-benzenacyclohexaphane} [( $\left.\left.S_{p}\right)-3 a\right]:$

Yellow solid, $12.2 \mathrm{mg}, 41 \%$ yield $(\mathrm{PE} / \mathrm{EA}=4 / 1)$. Analytical data for $\left(\boldsymbol{S}_{\boldsymbol{p}}\right)-3 \mathrm{a}: \mathrm{mp}$ : 248-250 ${ }^{\circ} \mathrm{C} ;{ }^{1} \mathrm{H}$ NMR $\left(500 \mathrm{MHz}, \mathrm{CDCl}_{3}\right) \delta 8.49(\mathrm{~d}, J=6.8 \mathrm{~Hz}, 1 \mathrm{H}), 7.79(\mathrm{~d}, J=9.2$ $\mathrm{Hz}, 1 \mathrm{H}), 7.46-7.37(\mathrm{~m}, 1 \mathrm{H}), 6.89(\mathrm{t}, J=6.7 \mathrm{~Hz}, 1 \mathrm{H}), 6.79(\mathrm{~d}, J=7.5 \mathrm{~Hz}, 1 \mathrm{H}), 6.65(\mathrm{~d}$, $J=7.5 \mathrm{~Hz}, 1 \mathrm{H}), 6.47-6.29(\mathrm{~m}, 2 \mathrm{H}), 5.97(\mathrm{~d}, J=7.9 \mathrm{~Hz}, 1 \mathrm{H}), 5.10(\mathrm{dd}, J=7.8,1.7 \mathrm{~Hz}$, $1 \mathrm{H}), 3.96-3.87(\mathrm{~m}, 1 \mathrm{H}), 3.81(\mathrm{dd}, J=14.5,9.4 \mathrm{~Hz}, 1 \mathrm{H}), 3.28-3.20(\mathrm{~m}, 1 \mathrm{H}), 3.15-3.05$ $(\mathrm{m}, 2 \mathrm{H}), 3.03-2.94(\mathrm{~m}, 2 \mathrm{H}), 2.74-2.65(\mathrm{~m}, 1 \mathrm{H}) ;{ }^{13} \mathrm{C} \mathrm{NMR}\left(126 \mathrm{MHz}, \mathrm{CDCl}_{3}\right) \delta 138.7$, $136.6,132.5,132.2,130.4,130.1,129.0,128.1,126.7,126.3,125.5,124.5,118.5$, 110.8, 34.5, 33.7, 33.3, 31.1; IR (neat) 2923, 1633, 1498, 1357, 1319, 1258, 1149, 
750, $516 \mathrm{~cm}^{-1}$; HRMS (APCI) m/z: calcd for $\mathrm{C}_{21} \mathrm{H}_{19} \mathrm{~N}_{2}[\mathrm{M}+\mathrm{H}]^{+}, 299.1543$; found, 299.1562. $[\alpha]_{\mathrm{D}}{ }^{20}=R_{p} / S_{p}-102.4 /+105.1\left(\mathrm{c} 0.25, \mathrm{CHCl}_{3}\right)$.

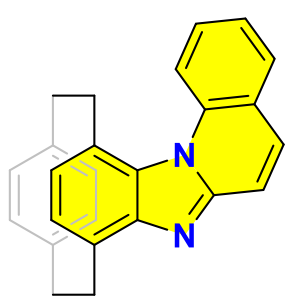

$$
\left(S_{p}\right)-3 b
$$

$\left(S_{p}\right)$-1(8,11)-benzo[4,5]imidazo[1,2-a]quinolina-4(1,4)-benzenacyclohexaphane $\left[\left(S_{p}\right)-3 b\right]:$

Yellow solid, $22.6 \mathrm{mg}$, 64\% yield $(\mathrm{PE} / \mathrm{EA}=4 / 1)$. Analytical data for $\left(\boldsymbol{S}_{\boldsymbol{p}}\right)-3 \mathbf{b}$ : $\mathrm{mp}$ : 252-254 ${ }^{\circ} \mathrm{C} ;{ }^{1} \mathrm{H}$ NMR $\left(500 \mathrm{MHz}, \mathrm{CDCl}_{3}\right) \delta 7.94(\mathrm{~d}, J=8.3 \mathrm{~Hz}, 1 \mathrm{H}), 7.87(\mathrm{~d}, J=7.6$ $\mathrm{Hz}, 1 \mathrm{H}), 7.76-7.65(\mathrm{~m}, 3 \mathrm{H}), 7.49(\mathrm{t}, J=7.4 \mathrm{~Hz}, 1 \mathrm{H}), 6.86-6.77(\mathrm{~m}, 2 \mathrm{H}), 6.50(\mathrm{~d}, J=$ $6.9 \mathrm{~Hz}, 1 \mathrm{H}), 6.43(\mathrm{~d}, J=7.6 \mathrm{~Hz}, 1 \mathrm{H}), 6.20(\mathrm{~d}, J=7.5 \mathrm{~Hz}, 1 \mathrm{H}), 5.11(\mathrm{~d}, J=7.6 \mathrm{~Hz}$, $1 \mathrm{H})$, 4.02-3.92 (m, 1H), 3.73-3.64 (m, 1H), 3.26-3.17 (m, 2H), 3.07-2.97 (m, 2H), 2.87-2.80 (m, 1H), 2.08-2.00 (m, 1H); $\left.{ }^{13} \mathrm{C} \mathrm{NMR} \mathrm{(126} \mathrm{MHz,} \mathrm{CDCl}_{3}\right) \delta$ 147.0, 138.6, 137.2 , 134.5, 132.1, 131.9, 131.4, 130.9, 130.6, 130.5, 130.4, 128.7, 128.6, 127.3, 126.9, 124.7, 124.2, 123.7, 118.1, 117.8, 36.7, 34.5, 34.2, 30.9; IR (neat) 2926, 1605, 1531, 1445, 1388, 1324, 1068, 808, 755, $513 \mathrm{~cm}^{-1}$; HRMS (APCI) m/z: calcd for $\mathrm{C}_{25} \mathrm{H}_{21} \mathrm{~N}_{2}[\mathrm{M}+\mathrm{H}]^{+}, 349.1699$; found, 349.1708. $[\alpha]_{\mathrm{D}}{ }^{20}=R_{p} / S_{p^{-}}-369.6 /+392.4$ (c 0.25 , $\left.\mathrm{CHCl}_{3}\right)$.

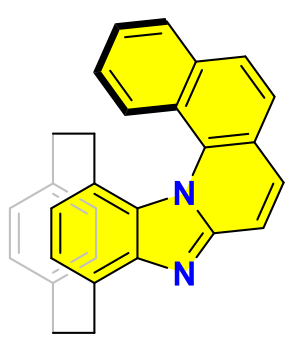

$\left(S_{p}, P\right)-3 c$

$\left(S_{p}, P\right)-1(10,13)-b e n z o[h]$ benzo[4,5]imidazo[1,2-a]quinolina-4(1,4)-benzena-cyclo h-xaphane $\left[\left(S_{p}, P\right)-3 c\right]$ :

Yellow solid, $23.8 \mathrm{mg}$, 59\% yield (PE/EA=4/1). Analytical data for $\left(\boldsymbol{S}_{\boldsymbol{p}}, \boldsymbol{P}\right)-\mathbf{3 c}$ : $\mathrm{mp}$ : 236-238 ${ }^{\circ} \mathrm{C}$; ${ }^{1} \mathrm{H}$ NMR $\left(500 \mathrm{MHz}, \mathrm{CDCl}_{3}\right) \delta 8.74(\mathrm{~d}, J=8.6 \mathrm{~Hz}, 1 \mathrm{H}), 8.00(\mathrm{~d}, J=8.0$ $\mathrm{Hz}, 1 \mathrm{H}), 7.94-7.88(\mathrm{~m}, 2 \mathrm{H}), 7.87-7.79(\mathrm{~m}, 2 \mathrm{H}), 7.61(\mathrm{t}, J=7.3 \mathrm{~Hz}, 1 \mathrm{H}), 7.37(\mathrm{t}, J=$ $7.6 \mathrm{~Hz}, 1 \mathrm{H}), 6.83(\mathrm{~d}, J=7.5 \mathrm{~Hz}, 1 \mathrm{H}), 6.68(\mathrm{~d}, J=7.5 \mathrm{~Hz}, 1 \mathrm{H}), 6.40(\mathrm{~d}, J=7.1 \mathrm{~Hz}$, $1 \mathrm{H}), 6.26(\mathrm{~d}, J=7.2 \mathrm{~Hz}, 1 \mathrm{H}), 6.21(\mathrm{~d}, J=7.1 \mathrm{~Hz}, 1 \mathrm{H}), 4.78(\mathrm{~d}, J=6.9 \mathrm{~Hz}, 1 \mathrm{H})$, 4.05-3.95 (m, 1H), 3.30-3.17 (m, 1H), 3.08-2.94 (m, 2H), 2.58-2.40 (m, 3H), 2.15 (s, $1 \mathrm{H}) ;{ }^{13} \mathrm{C}$ NMR $\left(126 \mathrm{MHz}, \mathrm{CDCl}_{3}\right) \delta 148.5,147.2,138.2,137.5,134.6,133.0,132.1$, $131.9,131.9,130.6,130.2,129.9,129.7,129.6,129.4,128.4,127.9,127.8,127.1$, $125.8,125.3,125.2,125.1,124.8,124.4,124.3,122.0,121.7,118.1,36.4,34.5$, 34.3, 30.8; IR 2919, 1499, 1393, 1314, 1131, 828, 751, $647 \mathrm{~cm}^{-1}$; HRMS (APCI) m/ z: calcd for $\mathrm{C}_{29} \mathrm{H}_{23} \mathrm{~N}_{2}[\mathrm{M}+\mathrm{H}]^{+}, 399.1856$; found, 399.1861 . 
$[\alpha]_{\mathrm{D}}^{20}=R_{p} / S_{p}-906.4 /+996.8\left(\mathrm{c} 0.25, \mathrm{CHCl}_{3}\right)$. 


\section{References}

[1] (a) Masters, K.-S.; Rauws, T. R. M.; Yadav, A. K.; Herrebout, W. A.; der Veken, B. V.; Maes, B. U. W. On the Importance of an Acid Additive in the Synthesis of Pyrido[1,2-a]benzimidazoles by Direct Copper-Catalyzed Amination. Chem. - Eur. J. 2011, 17, 6315. (b) Rao, D. N.; Rasheed, Sk.; Raina, G.; Ahmed, Q. N.; Jaladanki, C. K.; Bharatam, P. V.; Das, P. Cobalt-Catalyzed Regioselective Ortho C( $\left.\mathrm{sp}^{2}\right)-\mathrm{H}$ Bond Nitration of Aromatics through Proton-Coupled Electron Transfer Assistance. $J$. Org. Chem. 2017, 82, 7234.

[2] Otani, T.; Tsuyuki, A.; Iwachi, T.; Someya, S.; Tateno, K.; Kawai, H.; Saito, T.; Kanyiva, K. S.; Shibata, T. Facile Two-Step Synthesis of 1,10-Phenanthroline-Derived Polyaza[7]helicenes with High Fluorescence and CPL Efficiency. Angew. Chem., Int. Ed. 2017, 56, 3906.

[3] He, Y.; Huang, J.; Liang, D.; Liu, L.; Zhu, Q. C-H cycloamination of $\mathrm{N}$-aryl-2-aminopyridines and $\mathrm{N}$-arylamidines catalyzed by an in situ generated hypervalent iodine(III) reagent. Chem. Commun. 2013, 49, 7352. 
Theoretical Calculations:

3a Total Energy (B3LYP/6-31G): - 920.315535 a.u. Imaginary frequencies: 0

\begin{tabular}{|c|c|c|c|}
\hline $3 \mathbf{a}$ & $X$ & $\mathrm{Y}$ & $\mathrm{Z}$ \\
\hline $\mathrm{C}$ & -2.66568 & 0.100219 & -2.29498 \\
\hline $\mathrm{C}$ & -2.70294 & -1.20447 & -1.7929 \\
\hline $\mathrm{C}$ & -1.54863 & -1.78909 & -1.24343 \\
\hline $\mathrm{C}$ & -0.32181 & -1.14731 & -1.49206 \\
\hline $\mathrm{C}$ & -0.28396 & 0.156416 & -1.99842 \\
\hline $\mathrm{C}$ & -1.47687 & 0.851825 & -2.26228 \\
\hline $\mathrm{C}$ & -1.5221 & 2.368011 & -2.22074 \\
\hline $\mathrm{C}$ & -1.64229 & -2.86917 & -0.17943 \\
\hline $\mathrm{C}$ & -3.0992 & 1.31056 & 0.589396 \\
\hline $\mathrm{C}$ & -3.21008 & -0.00718 & 1.089575 \\
\hline $\mathrm{C}$ & -2.09327 & -0.8072 & 1.36372 \\
\hline $\mathrm{C}$ & -0.87324 & -0.09956 & 1.402441 \\
\hline $\mathrm{C}$ & -0.74368 & 1.221008 & 0.876633 \\
\hline $\mathrm{C}$ & -1.85895 & 1.891191 & 0.326572 \\
\hline $\mathrm{C}$ & -1.65703 & 2.962241 & -0.72268 \\
\hline $\mathrm{C}$ & -2.2111 & -2.31713 & 1.218967 \\
\hline $\mathrm{N}$ & 0.43934 & -0.49651 & 1.6974 \\
\hline $\mathrm{C}$ & 1.286733 & 0.602227 & 1.339331 \\
\hline $\mathrm{N}$ & 0.587315 & 1.620751 & 0.833391 \\
\hline $\mathrm{C}$ & 0.949981 & -1.6477 & 2.26157 \\
\hline $\mathrm{C}$ & 2.298855 & -1.75982 & 2.46902 \\
\hline $\mathrm{C}$ & 3.177552 & -0.68756 & 2.116646 \\
\hline $\mathrm{C}$ & 2.680138 & 0.470978 & 1.568506 \\
\hline $\mathrm{H}$ & -3.59436 & 0.583462 & -2.58762 \\
\hline $\mathrm{H}$ & -3.65993 & -1.71227 & -1.70174 \\
\hline $\mathrm{H}$ & 0.602132 & -1.61556 & -1.16245 \\
\hline $\mathrm{H}$ & 0.6639 & 0.684255 & -2.04097 \\
\hline $\mathrm{H}$ & -2.37487 & 2.732483 & -2.8055 \\
\hline $\mathrm{H}$ & -0.61686 & 2.794378 & -2.66724 \\
\hline $\mathrm{H}$ & -0.64604 & -3.29456 & -0.00947 \\
\hline $\mathrm{H}$ & -2.29424 & -3.69591 & -0.48871 \\
\hline $\mathrm{H}$ & -4.00136 & 1.806751 & 0.242105 \\
\hline $\mathrm{H}$ & -4.19101 & -0.47415 & 1.091063 \\
\hline $\mathrm{H}$ & -2.49646 & 3.666762 & -0.7157 \\
\hline $\mathrm{H}$ & -0.74539 & 3.522007 & -0.49707 \\
\hline $\mathrm{H}$ & -3.275 & -2.57187 & 1.277452 \\
\hline $\mathrm{H}$ & -1.7227 & -2.87639 & 2.02668 \\
\hline $\mathrm{H}$ & 0.243582 & -2.41925 & 2.528462 \\
\hline $\mathrm{H}$ & 2.692019 & -2.66697 & 2.910681 \\
\hline $\mathrm{H}$ & 4.242065 & -0.79406 & 2.292371 \\
\hline
\end{tabular}




\begin{tabular}{llll}
\hline $\mathrm{H}$ & 3.314239 & 1.30577 & 1.299007 \\
\hline
\end{tabular}

3b Total Energy (B3LYP/6-31G): -1073.926130 a.u. Imaginary frequencies: 0

\begin{tabular}{|c|c|c|c|}
\hline $3 b$ & $X$ & $\mathrm{Y}$ & $\mathrm{Z}$ \\
\hline $\mathrm{C}$ & -2.64328 & 0.475895 & -3.17215 \\
\hline $\mathrm{C}$ & -2.68636 & -0.82693 & -2.66655 \\
\hline $\mathrm{C}$ & -1.53553 & -1.40995 & -2.10833 \\
\hline $\mathrm{C}$ & -0.3059 & -0.77255 & -2.35186 \\
\hline $\mathrm{C}$ & -0.26121 & 0.526355 & -2.86796 \\
\hline $\mathrm{C}$ & -1.45152 & 1.222442 & -3.13834 \\
\hline $\mathrm{C}$ & -1.49545 & 2.737182 & -3.08008 \\
\hline $\mathrm{C}$ & -1.63213 & -2.48691 & -1.04503 \\
\hline $\mathrm{C}$ & -3.02577 & 1.687912 & -0.26456 \\
\hline $\mathrm{C}$ & -3.16909 & 0.376932 & 0.23516 \\
\hline $\mathrm{C}$ & -2.07776 & -0.45257 & 0.546964 \\
\hline $\mathrm{C}$ & -0.84415 & 0.236921 & 0.635819 \\
\hline $\mathrm{C}$ & -0.6797 & 1.5258 & 0.036507 \\
\hline $\mathrm{C}$ & -1.76498 & 2.225827 & -0.5282 \\
\hline $\mathrm{C}$ & -1.52497 & 3.299864 & -1.56894 \\
\hline $\mathrm{C}$ & -2.25106 & -1.95395 & 0.332698 \\
\hline $\mathrm{N}$ & 0.463432 & -0.16077 & 0.985228 \\
\hline $\mathrm{C}$ & 1.33366 & 0.854074 & 0.495797 \\
\hline $\mathrm{N}$ & 0.668915 & 1.859767 & -0.06456 \\
\hline $\mathrm{C}$ & 0.941117 & -1.18722 & 1.817491 \\
\hline $\mathrm{C}$ & 2.35429 & -1.37632 & 1.884847 \\
\hline $\mathrm{C}$ & 3.235721 & -0.43751 & 1.228934 \\
\hline $\mathrm{C}$ & 2.74367 & 0.669149 & 0.605468 \\
\hline $\mathrm{C}$ & 0.094976 & -1.95632 & 2.636022 \\
\hline $\mathrm{C}$ & 0.623002 & -2.97666 & 3.424228 \\
\hline $\mathrm{C}$ & 2.003685 & -3.23877 & 3.425194 \\
\hline $\mathrm{C}$ & 2.854072 & -2.43238 & 2.677948 \\
\hline $\mathrm{H}$ & -3.56926 & 0.962983 & -3.4673 \\
\hline $\mathrm{H}$ & -3.64449 & -1.33322 & -2.57875 \\
\hline $\mathrm{H}$ & 0.61374 & -1.24059 & -2.01034 \\
\hline $\mathrm{H}$ & 0.687459 & 1.052846 & -2.90438 \\
\hline $\mathrm{H}$ & -2.38928 & 3.102708 & -3.59909 \\
\hline $\mathrm{H}$ & -0.62631 & 3.17814 & -3.5817 \\
\hline $\mathrm{H}$ & -0.63071 & -2.88457 & -0.84326 \\
\hline $\mathrm{H}$ & -2.25414 & -3.33264 & -1.36632 \\
\hline $\mathrm{H}$ & -3.9121 & 2.20648 & -0.61912 \\
\hline $\mathrm{H}$ & -4.15769 & -0.07284 & 0.206178 \\
\hline $\mathrm{H}$ & -2.30491 & 4.068156 & -1.51252 \\
\hline $\mathrm{H}$ & -0.56486 & 3.781174 & -1.36548 \\
\hline $\mathrm{H}$ & -1.82878 & -2.57684 & 1.125728 \\
\hline
\end{tabular}




\begin{tabular}{cccc}
\hline $\mathrm{H}$ & -3.32873 & -2.1532 & 0.318009 \\
$\mathrm{H}$ & 4.305395 & -0.60591 & 1.29614 \\
$\mathrm{H}$ & 3.380475 & 1.426904 & 0.167761 \\
$\mathrm{H}$ & -0.95787 & -1.71832 & 2.687464 \\
$\mathrm{H}$ & -0.04067 & -3.55921 & 4.054503 \\
$\mathrm{H}$ & 2.405887 & -4.04241 & 4.032311 \\
$\mathrm{H}$ & 3.928325 & -2.58677 & 2.715525 \\
\hline
\end{tabular}

3c Total Energy (B3LYP/6-31G): -1227.524857 a.u. Imaginary frequencies: 0

\begin{tabular}{|c|c|c|c|}
\hline $3 c$ & $X$ & $\mathrm{Y}$ & $\mathrm{Z}$ \\
\hline $\mathrm{C}$ & -2.40716 & 0.889164 & -3.82368 \\
\hline $\mathrm{C}$ & -2.44633 & -0.42978 & -3.36066 \\
\hline $\mathrm{C}$ & -1.33375 & -0.98573 & -2.70566 \\
\hline $\mathrm{C}$ & -0.11317 & -0.29464 & -2.80828 \\
\hline $\mathrm{C}$ & -0.07135 & 1.020614 & -3.28151 \\
\hline $\mathrm{C}$ & -1.25658 & 1.679526 & -3.65027 \\
\hline $\mathrm{C}$ & -1.36676 & 3.189491 & -3.55887 \\
\hline $\mathrm{C}$ & -1.49083 & -2.09808 & -1.68677 \\
\hline $\mathrm{C}$ & -3.12377 & 2.001415 & -0.94667 \\
\hline $\mathrm{C}$ & -3.25722 & 0.666082 & -0.50746 \\
\hline $\mathrm{C}$ & -2.16652 & -0.12049 & -0.10698 \\
\hline $\mathrm{C}$ & -0.98226 & 0.615734 & 0.139551 \\
\hline $\mathrm{C}$ & -0.81378 & 1.925045 & -0.40927 \\
\hline $\mathrm{C}$ & -1.86917 & 2.599914 & -1.05938 \\
\hline $\mathrm{C}$ & -1.57151 & 3.710379 & -2.04593 \\
\hline $\mathrm{C}$ & -2.23034 & -1.6237 & -0.34428 \\
\hline $\mathrm{N}$ & 0.306029 & 0.241432 & 0.589603 \\
\hline $\mathrm{C}$ & 1.182037 & 1.287148 & 0.158467 \\
\hline $\mathrm{N}$ & 0.525954 & 2.296917 & -0.406 \\
\hline $\mathrm{C}$ & 0.790106 & -0.83172 & 1.366292 \\
\hline $\mathrm{C}$ & 2.183884 & -1.07305 & 1.304084 \\
\hline $\mathrm{C}$ & 3.065295 & -0.10104 & 0.707497 \\
\hline $\mathrm{C}$ & 2.587444 & 1.084356 & 0.233257 \\
\hline $\mathrm{C}$ & -0.01982 & -1.59148 & 2.289771 \\
\hline $\mathrm{C}$ & 0.55917 & -2.7624 & 2.895284 \\
\hline $\mathrm{C}$ & 1.92548 & -3.09209 & 2.646492 \\
\hline $\mathrm{C}$ & 2.719068 & -2.24307 & 1.923092 \\
\hline $\mathrm{C}$ & -1.31261 & -1.18667 & 2.732147 \\
\hline $\mathrm{C}$ & -2.02263 & -1.93517 & 3.652716 \\
\hline $\mathrm{C}$ & -1.48681 & -3.13639 & 4.173534 \\
\hline $\mathrm{C}$ & -0.21737 & -3.5331 & 3.80259 \\
\hline $\mathrm{H}$ & -3.31865 & 1.349116 & -4.1972 \\
\hline $\mathrm{H}$ & -3.38664 & -0.97505 & -3.38341 \\
\hline $\mathrm{H}$ & 0.787238 & -0.73801 & -2.39089 \\
\hline
\end{tabular}




\begin{tabular}{llll}
\hline $\mathrm{H}$ & 0.853966 & 1.58357 & -3.21007 \\
$\mathrm{H}$ & -0.46951 & 3.676087 & -3.95847 \\
$\mathrm{H}$ & -2.21722 & 3.536096 & -4.15771 \\
$\mathrm{H}$ & -2.06563 & -2.94388 & -2.08566 \\
$\mathrm{H}$ & -0.50044 & -2.48317 & -1.41776 \\
$\mathrm{H}$ & -3.99059 & 2.492813 & -1.37959 \\
$\mathrm{H}$ & -4.21301 & 0.172423 & -0.6595 \\
$\mathrm{H}$ & -0.65837 & 4.225156 & -1.73635 \\
$\mathrm{H}$ & -2.38574 & 4.444424 & -2.05192 \\
$\mathrm{H}$ & -3.28877 & -1.89655 & -0.42668 \\
$\mathrm{H}$ & -1.81807 & -2.21128 & 0.479026 \\
$\mathrm{H}$ & 4.130068 & -0.30887 & 0.708546 \\
$\mathrm{H}$ & 3.228495 & 1.86954 & -0.1455 \\
$\mathrm{H}$ & 2.337965 & -3.98801 & 3.099475 \\
$\mathrm{H}$ & 3.781155 & -2.43827 & 1.811681 \\
$\mathrm{H}$ & -1.74556 & -0.27232 & 2.352187 \\
$\mathrm{H}$ & -2.9993 & -1.59392 & 3.979875 \\
$\mathrm{H}$ & -2.06263 & -3.72682 & 4.878605 \\
$\mathrm{H}$ & 0.225719 & -4.42939 & 4.226814 \\
\hline
\end{tabular}

\section{X-ray Crystallographic Data for 3c}

Yellow crystal was grown by slow evaporation at room temperature from a solution in a mixture of hexane and ethyl acetate. The crystals were removed from the vial and covered with a layer of a viscous perfluoropolyether (FomblinY). Suitable crystals, selected with the aid of a microscope, were mounted on a cryoloop and placed in the low temperature nitrogen stream of the diffractometer. The intensity data sets were collected on a Bruker-Nonius KappaCCD diffractometer equipped with an Oxford Cryostream 700 unit. Crystallographic data for compounds $\mathbf{3 c}$ are presented in Table S1.

Table S1. Experimental data for the X-ray diffraction study on compound 3c:

\begin{tabular}{cc}
\hline Identification code & $\mathbf{3 c}$ \\
Empirical formula & $\mathrm{C}_{29} \mathrm{H}_{22} \mathrm{~N}_{2}$ \\
Formula weight & 398.48 \\
Temperature/K & $173(2)$ \\
Crystal system & orthorhombic \\
Space group & $\mathrm{P} 2{ }_{1} 2{ }_{1} 2_{1}$ \\
$\mathrm{a} / \AA$ & $7.4946(6)$ \\
$\mathrm{b} / \AA$ & $14.8178(12)$ \\
$\mathrm{c} / \AA$ & $18.1876(15)$ \\
$\alpha / /^{\circ}$ & 90 \\
$\beta /{ }^{\circ}$ & 90 \\
$\gamma^{\circ}$ & 90 \\
\hline
\end{tabular}




\begin{tabular}{|c|c|}
\hline Volume/Å3 & $2019.8(3)$ \\
\hline $\mathrm{Z}$ & 4 \\
\hline$\rho c a l c g / \mathrm{cm}^{3}$ & 1.310 \\
\hline$\mu / \mathrm{mm}^{-1}$ & 0.374 \\
\hline $\mathrm{F}(000)$ & 840.0 \\
\hline Crystal size $/ \mathrm{mm}^{3}$ & $0.150 \times 0.130 \times 0.090$ \\
\hline Radiation & $\operatorname{GaK} \alpha(\lambda=1.34139)$ \\
\hline $2 \Theta$ range for data collection ${ }^{\circ}$ & 6.694 to 107.946 \\
\hline Index ranges & $-9 \leq \mathrm{h} \leq 9,-17 \leq \mathrm{k} \leq 16,-21 \leq \mathrm{l} \leq 21$ \\
\hline Reflections collected & 28723 \\
\hline Independent reflections & $3688\left[\mathrm{R}_{\text {int }}=0.0779, \mathrm{R}_{\text {sigma }}=0.0396\right]$ \\
\hline Data/restraints/parameters & $3688 / 0 / 280$ \\
\hline Goodness-of-fit on $\mathrm{F}^{2}$ & 1.059 \\
\hline Final $\mathrm{R}$ indexes $[\mathrm{I}>=2 \sigma(\mathrm{I})]$ & $\mathrm{R}_{1}=0.0338, \mathrm{wR}_{2}=0.0861$ \\
\hline Final $\mathrm{R}$ indexes [all data] & $\mathrm{R}_{1}=0.0353, \mathrm{wR}_{2}=0.0876$ \\
\hline Largest diff. peak/hole / e $\AA^{-3}$ & $0.17 /-0.20$ \\
\hline Flack parameter & $0.5(3)$ \\
\hline CCDC code & 1956645 \\
\hline
\end{tabular}

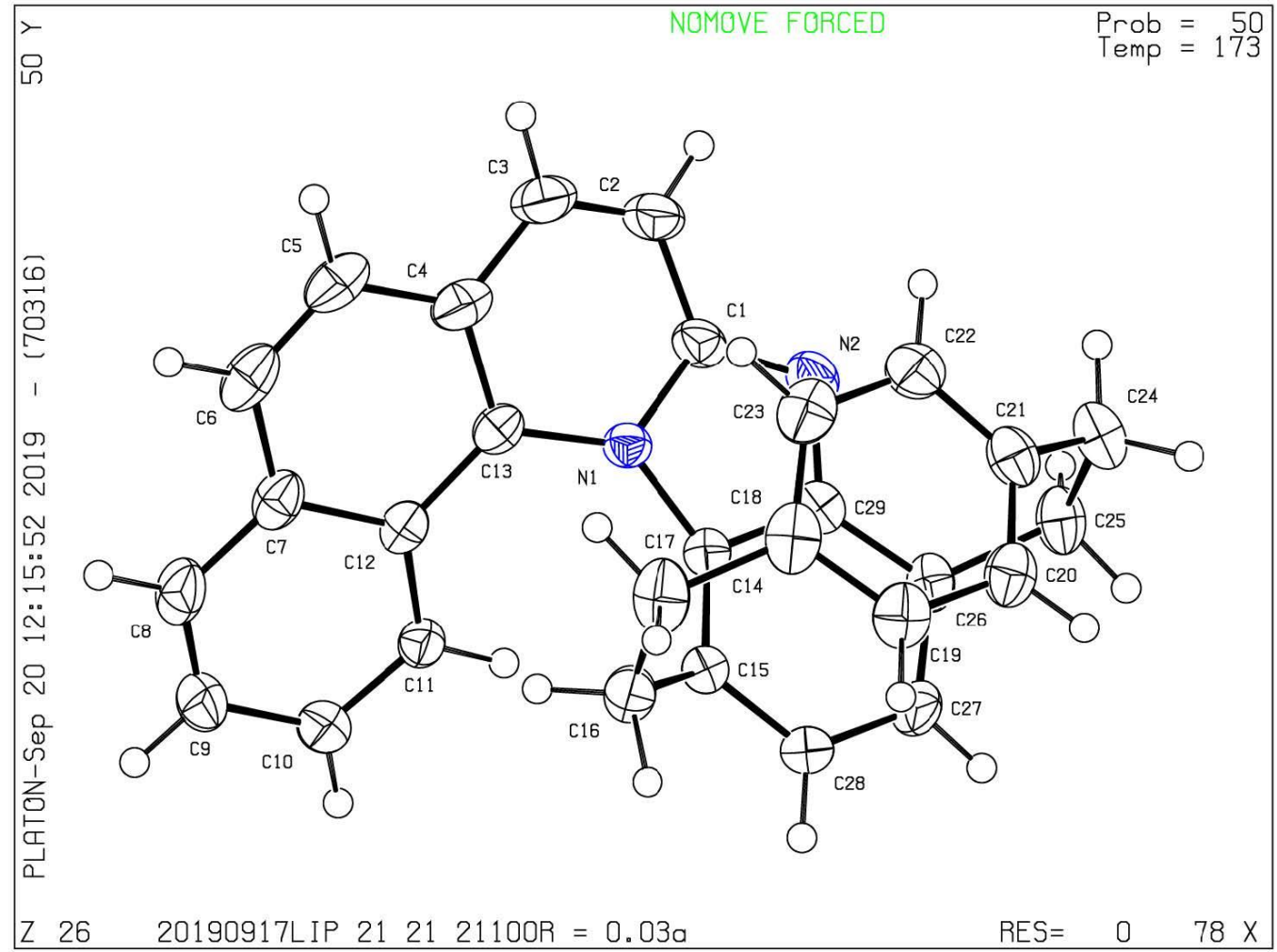

Figure S1. X-ray structure and numbering scheme for 3c. Thermal ellipsoids are drawn at the $50 \%$ probability level. 


\section{NMR and HRMS Spectra}

\section{${ }^{1} \mathrm{H}$ NMR of $2 \mathrm{a}\left(500 \mathrm{MHz}, \mathrm{CDCl}_{3}\right)$}

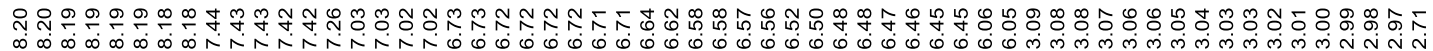

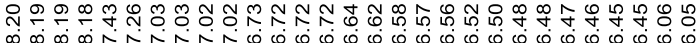

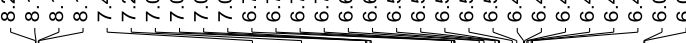

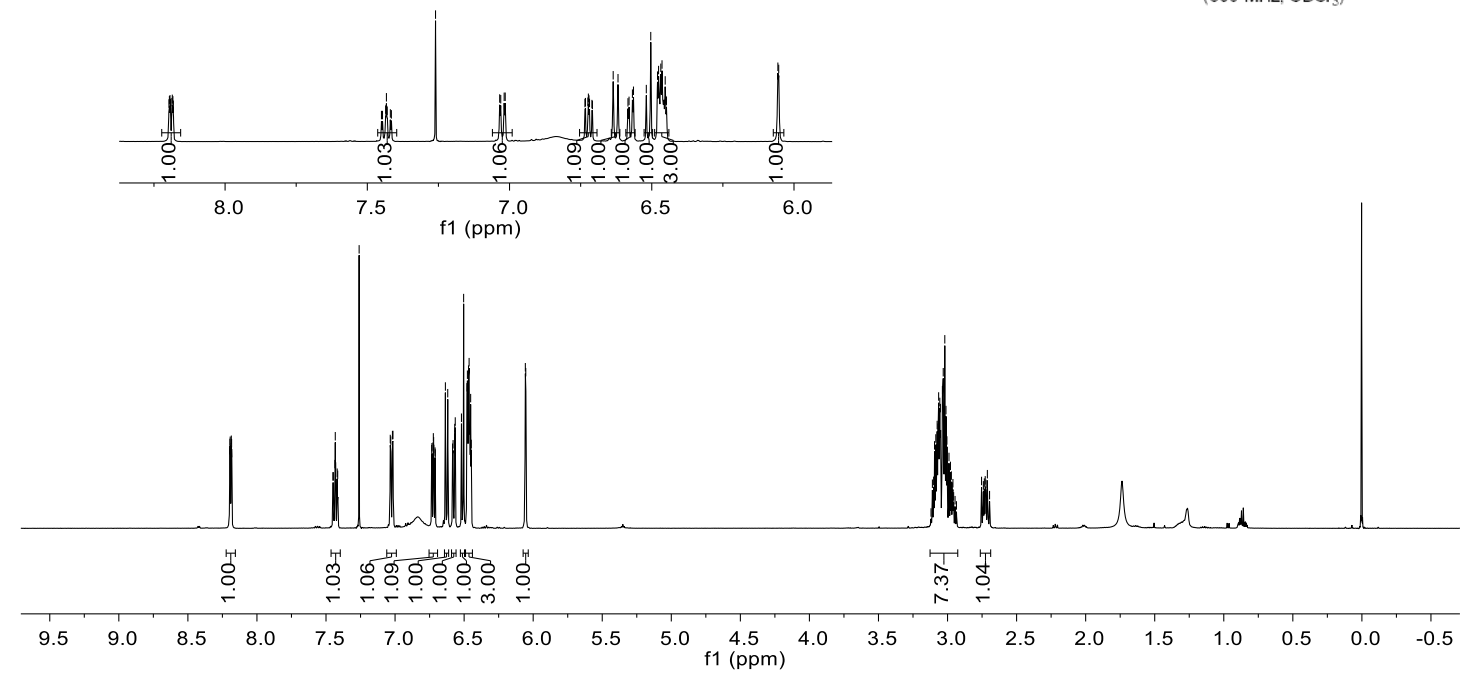

$\left.500 \mathrm{MHz}^{\mathrm{CDC}} \mathrm{C}_{3}\right)$

${ }^{13} \mathrm{C}$ NMR of 2a (126MHz, $\left.\mathrm{CDCl}_{3}\right)$

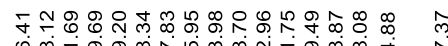

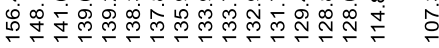

苟公各命

फि ले में

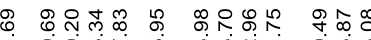

+

$\rightarrow 1$

I
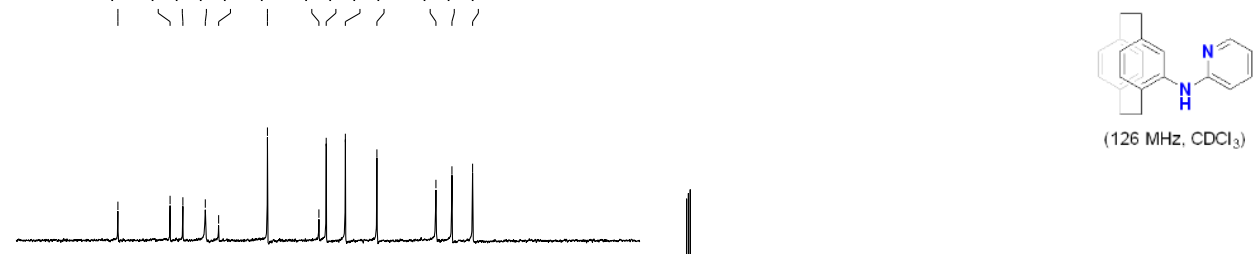

$\left(126 \mathrm{MHz}_{2} \mathrm{CDCl}_{3}\right)$

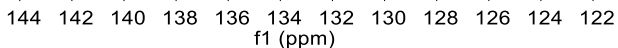
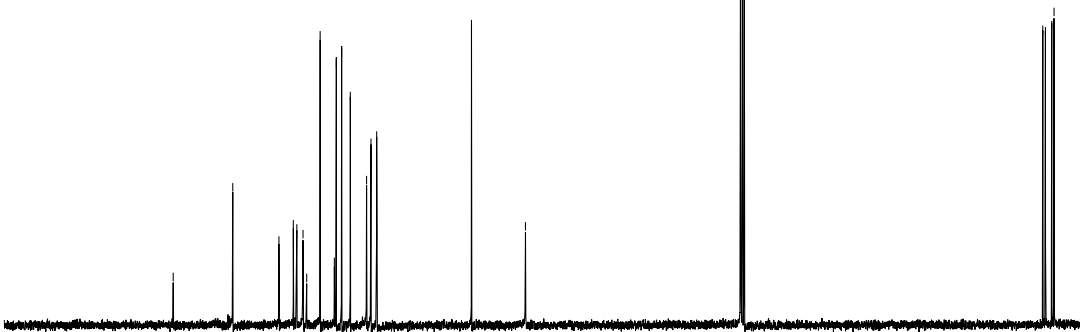

$\begin{array}{lllllllllllllllllllllllllll}80 & 170 & 160 & 150 & 140 & 130 & 120 & 110 & 100 & 90 & 80 & 70 & 60 & 50 & 40 & 30 & 20 & 10 & 0 & -10 & -:\end{array}$ 


\section{HRMS of 2a}

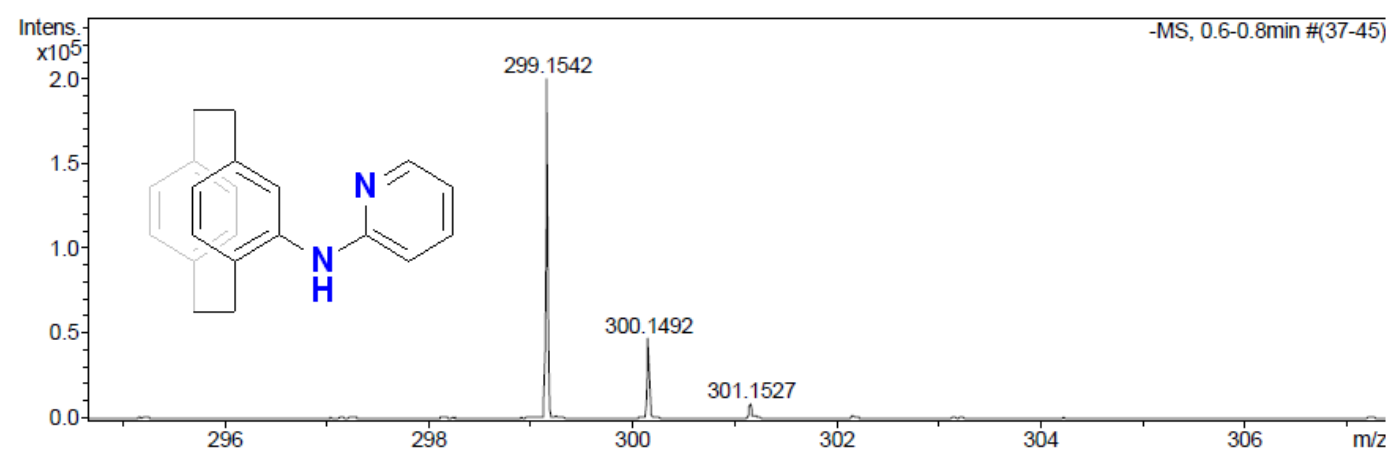


${ }^{1} \mathrm{H}$ NMR of $2 \mathrm{~b}\left(500 \mathrm{MHz}, \mathrm{CDCl}_{3}\right)$

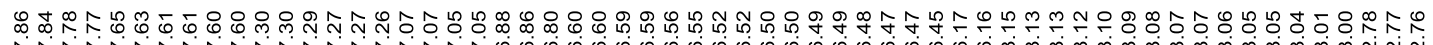

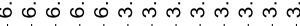

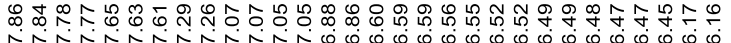

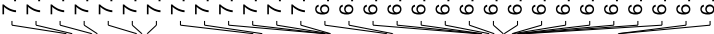

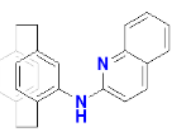

$\left(500 \mathrm{MHz}, \mathrm{CDCl}_{3}\right)$
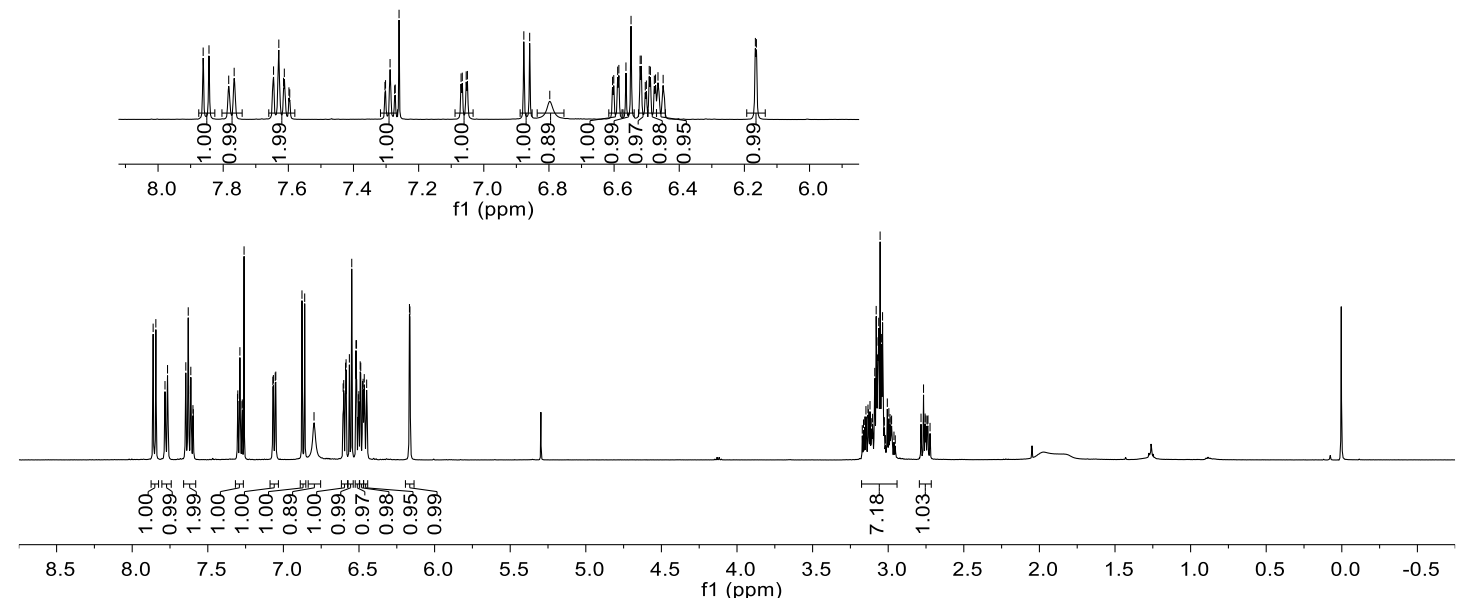

${ }^{13} \mathrm{C}$ NMR of $2 \mathrm{~b}\left(126 \mathrm{MHz}, \mathrm{CDCl}_{3}\right)$

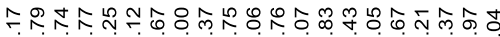

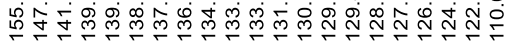

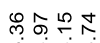

फं लं लंखें

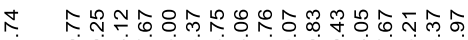

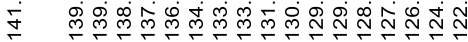

$\rightarrow$

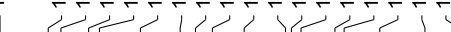
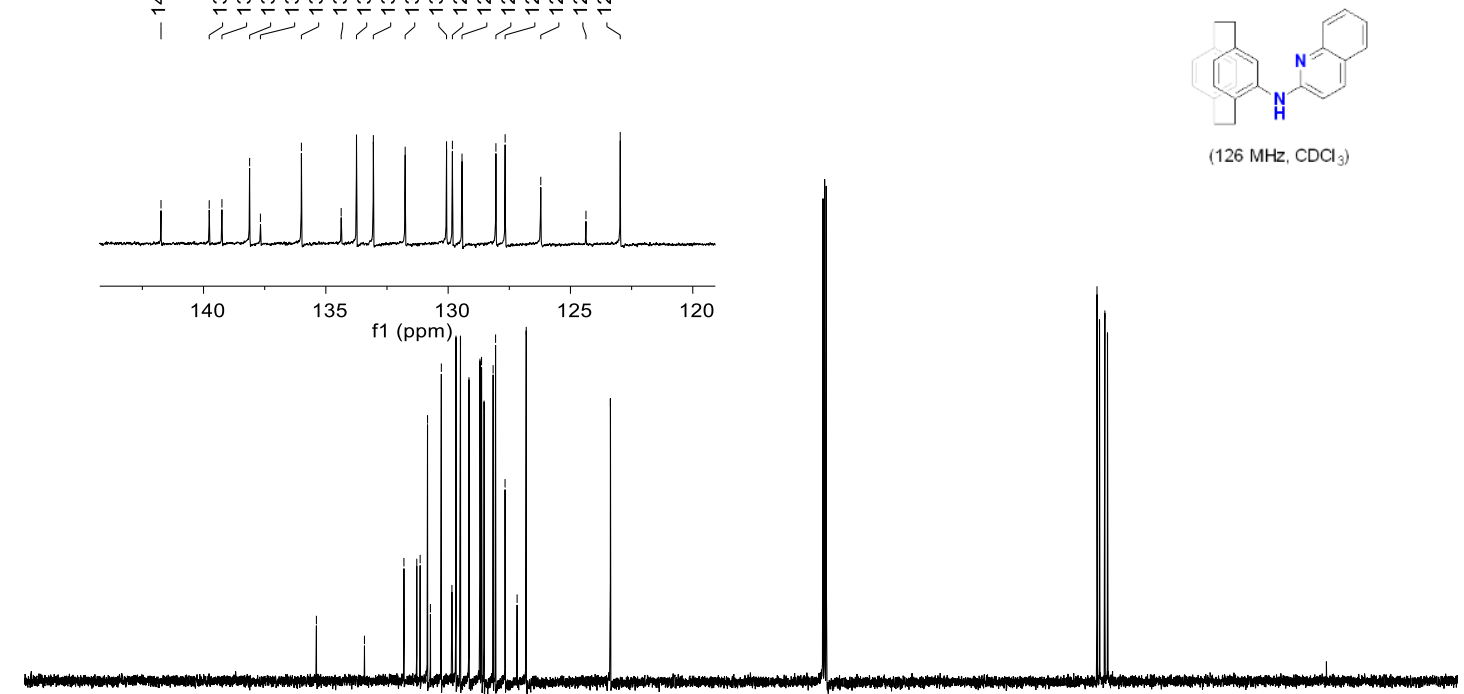

(126 MHz, $\mathrm{CDCl}_{3}$ )

$\begin{array}{llllllllllllllllllllllll}00 & 190 & 180 & 170 & 160 & 150 & 140 & 130 & 120 & 110 & 100 & 90 & 80 & 70 & 60 & 50 & 40 & 30 & 20 & 10 & 0 & -10 & -4\end{array}$ 


\section{HRMS of $\mathbf{2 b}$}

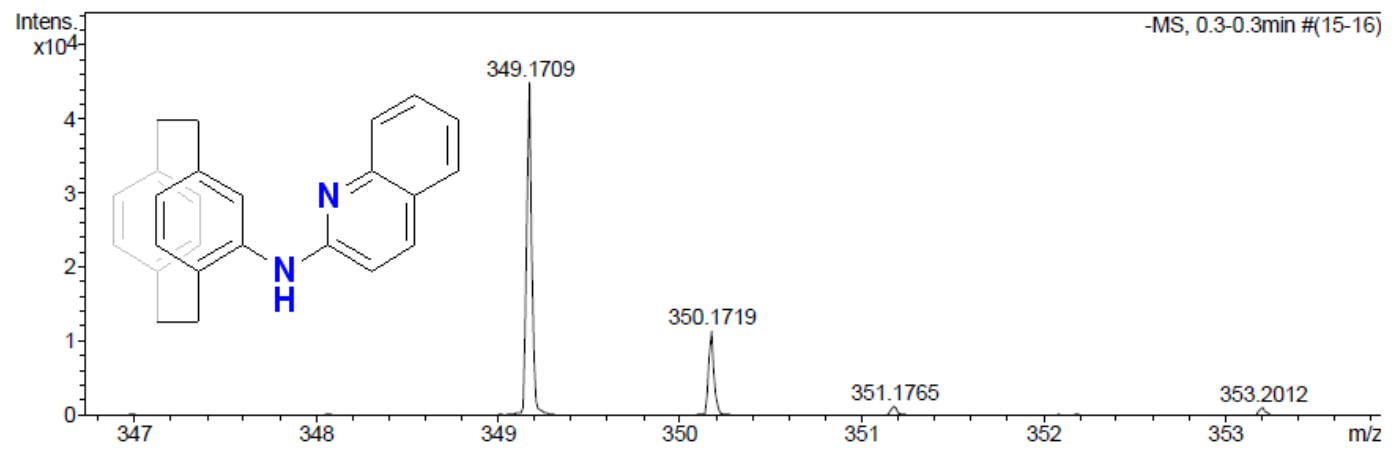




\section{${ }^{1} \mathrm{HNMR}$ of $2 \mathrm{c}\left(500 \mathrm{MHz}, \mathrm{CDCl}_{3}\right)$}

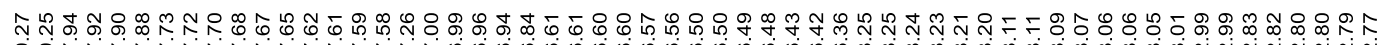
作

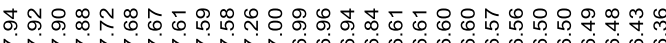

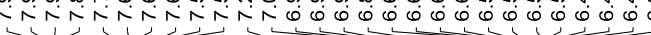

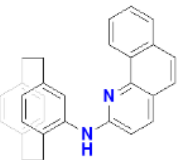

$\left(500 \mathrm{MHz}, \mathrm{CDCl}_{3}\right)$
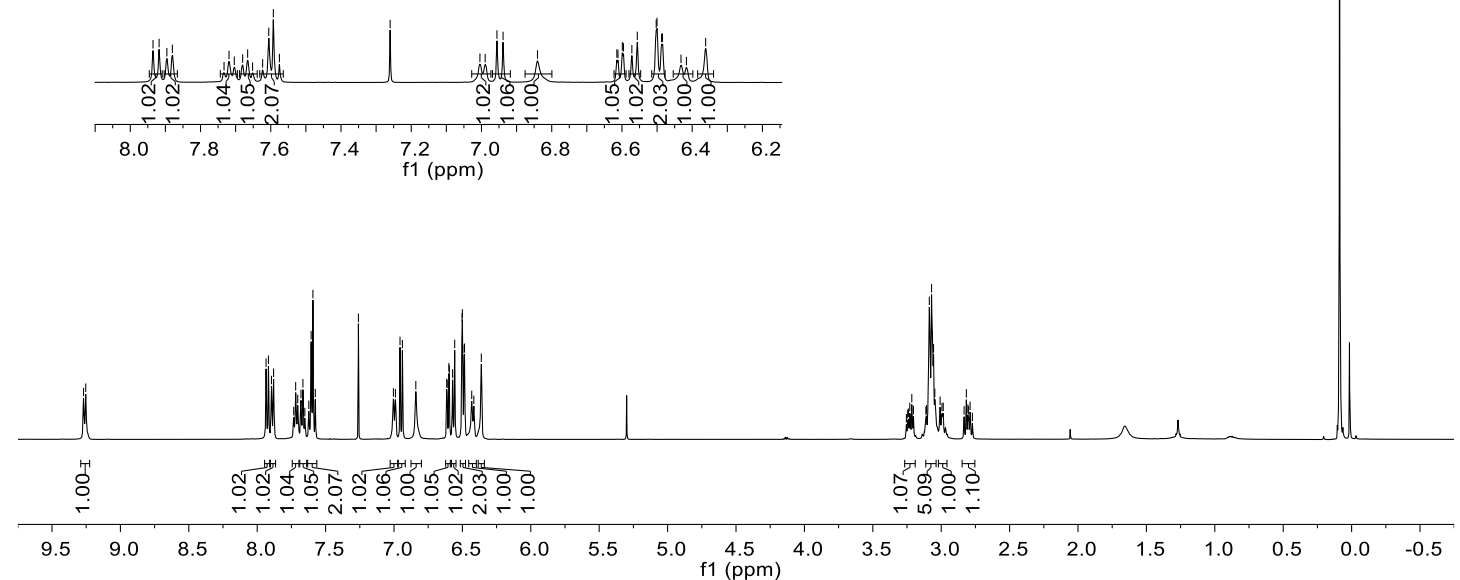

\section{${ }^{13} \mathrm{CNMR}$ of $2 \mathrm{c}\left(126 \mathrm{MHz}, \mathrm{CDCl}_{3}\right)$}

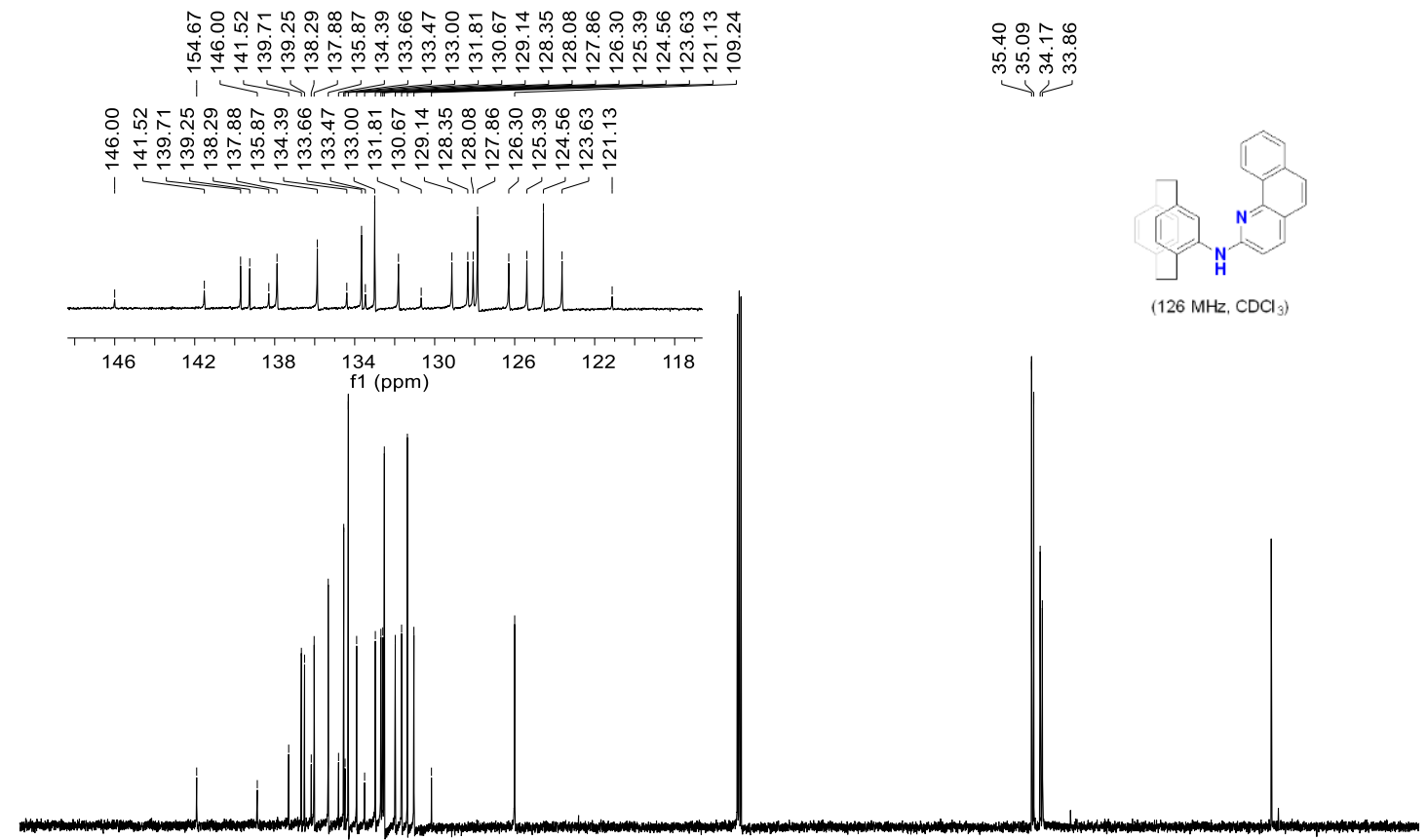

$\begin{array}{lllllllllll}80 & 170 & 160 & 150 & 140 & 130 & 120 & 110 & 100 & 90 & 80 \\ \mathrm{f} 1(\mathrm{ppm})\end{array}$ 


\section{HRMS of 2c}

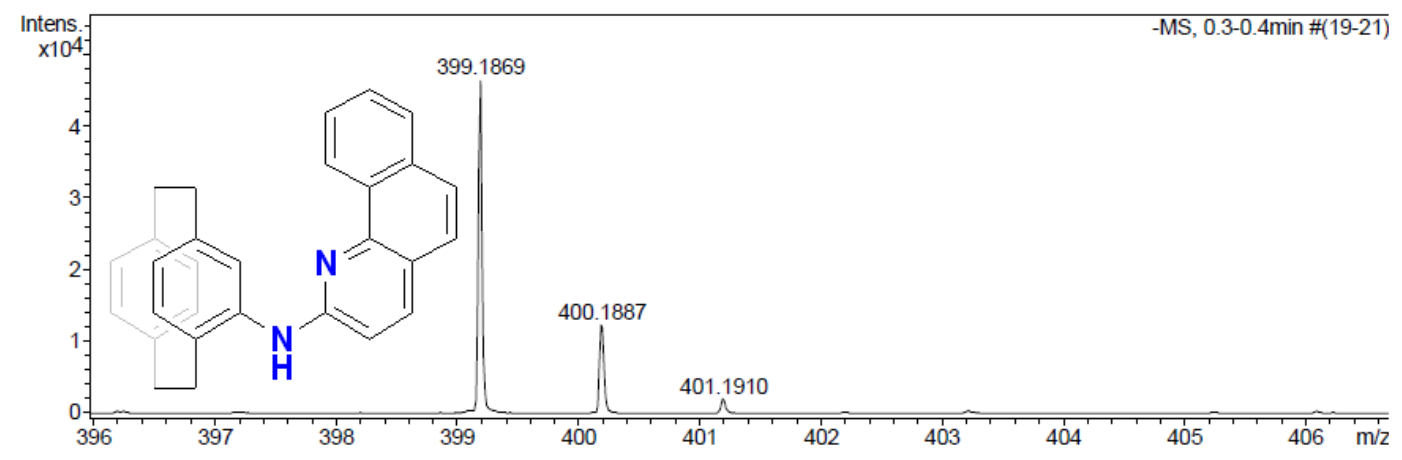


${ }^{1} \mathrm{H}$ NMR of $3 \mathrm{a}\left(500 \mathrm{MHz}, \mathrm{CDCl}_{3}\right)$

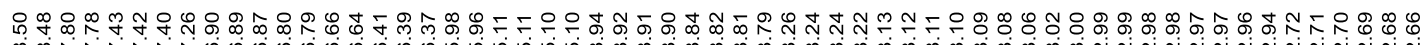

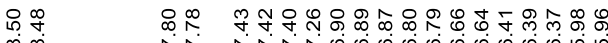

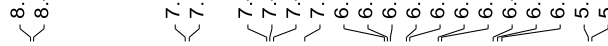

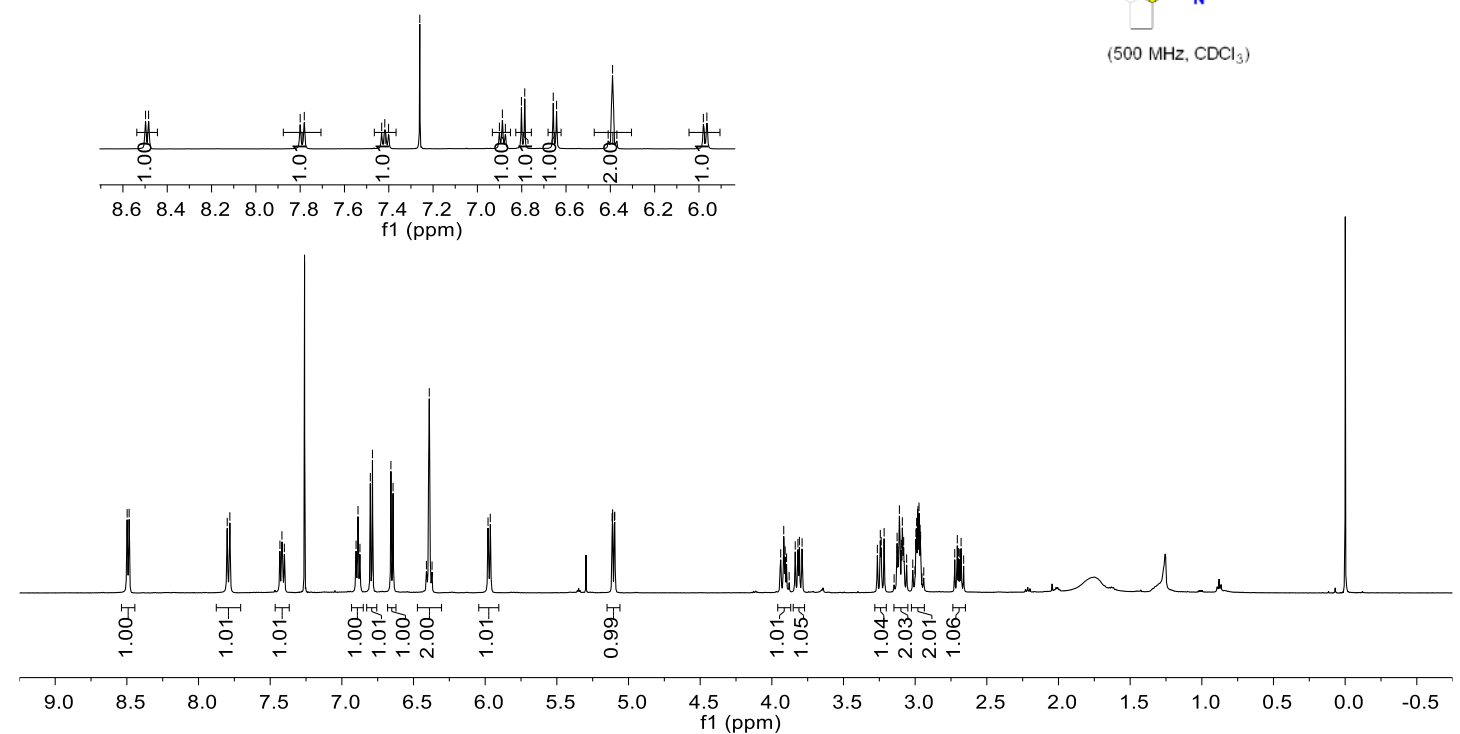

${ }^{13} \mathrm{C}$ NMR of $3 \mathrm{a}\left(126 \mathrm{MHz}, \mathrm{CDCl}_{3}\right)$

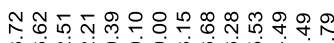

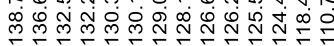

ํำ

प्लिल्लm

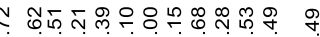

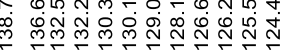

| । 1 ।

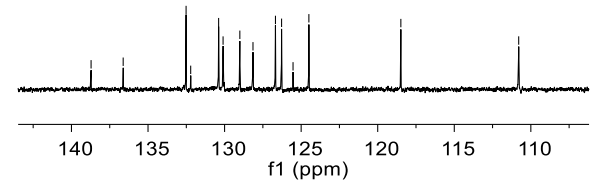

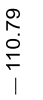

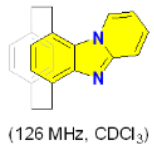

$\begin{array}{llllllll}170 & 160 & 150 & 140 & 130 & 120 & 110 & 100\end{array}$

$\stackrel{80}{\mathrm{f} 1}(\mathrm{ppm})$

$70 \quad 60 \quad 50$
0

$\begin{array}{lllllll}40 & 30 & 20 & 10 & 0 & -10 & -4\end{array}$ 


\section{HRMS of 3a}

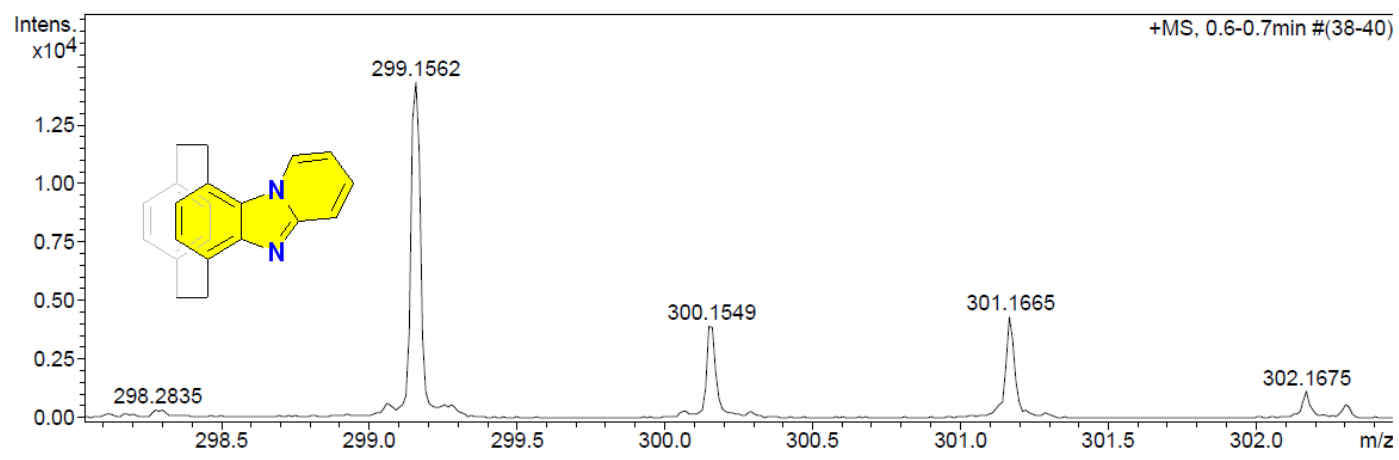


${ }^{1} \mathrm{H}$ NMR of $3 \mathrm{~b}\left(500 \mathrm{MHz}, \mathrm{CDCl}_{3}\right)$

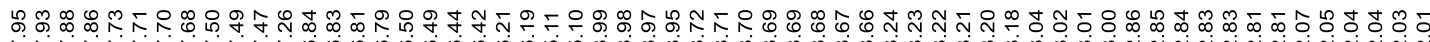

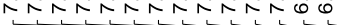

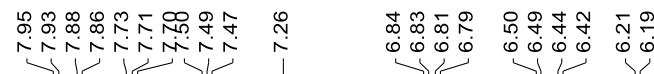
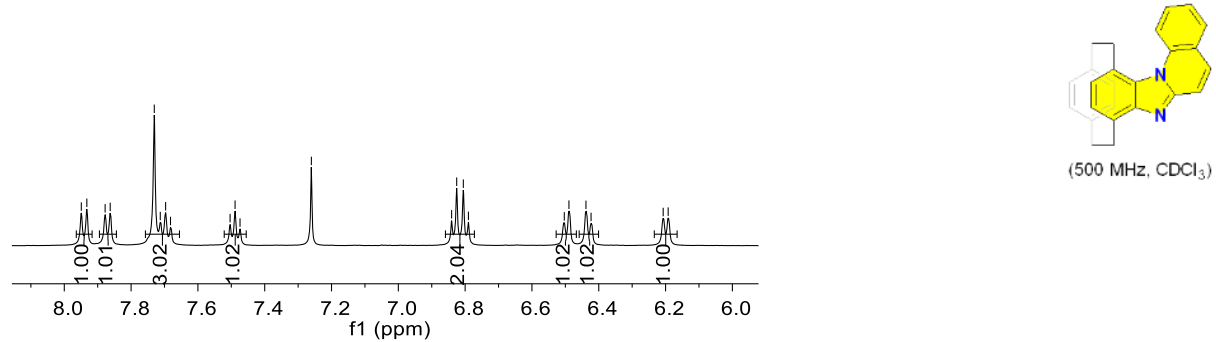

$\left(500 \mathrm{MHz}_{\mathrm{CDCl}}\right)$

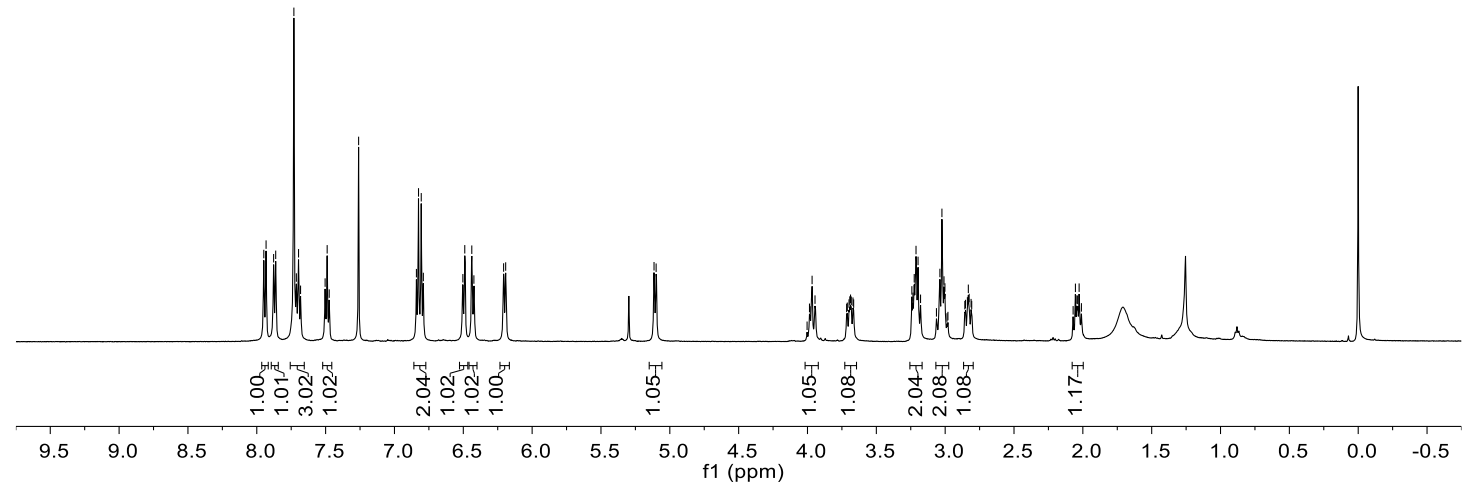

${ }^{13} \mathrm{C}$ NMR of $3 \mathrm{~b}\left(126 \mathrm{MHz}, \mathrm{CDCl}_{3}\right)$

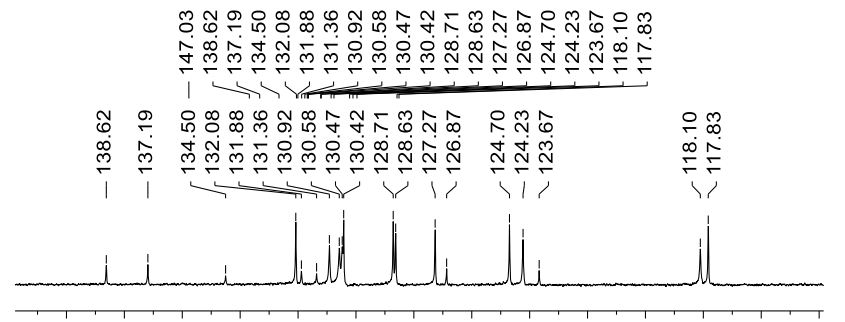

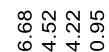

$\begin{array}{llllllllllllll}140 & 138 & 136 & 134 & 132 & 130 & 128 & 126 & 124 & 122 & 120 & 118 & 116 & 114\end{array}$

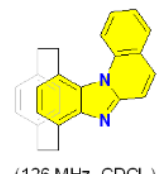

$\left(126 \mathrm{MHz} \mathrm{CDCl}_{3}\right)$

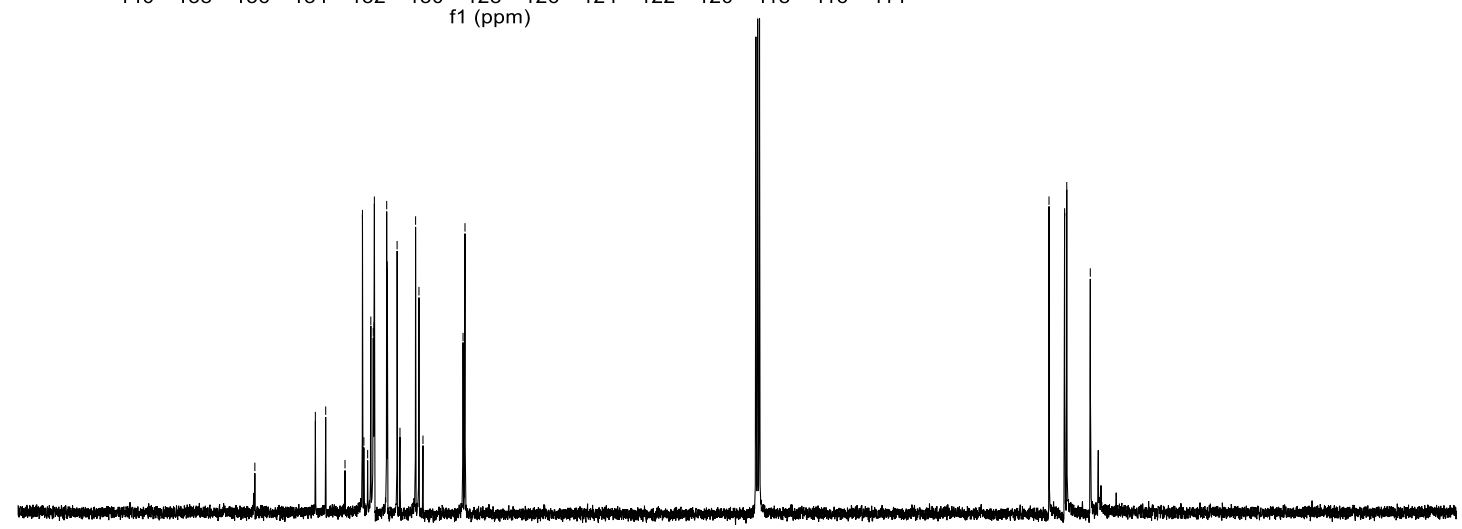

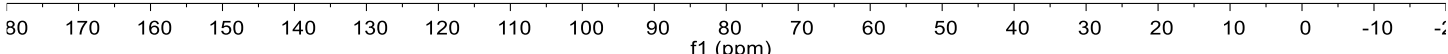




\section{HRMS of 3b}

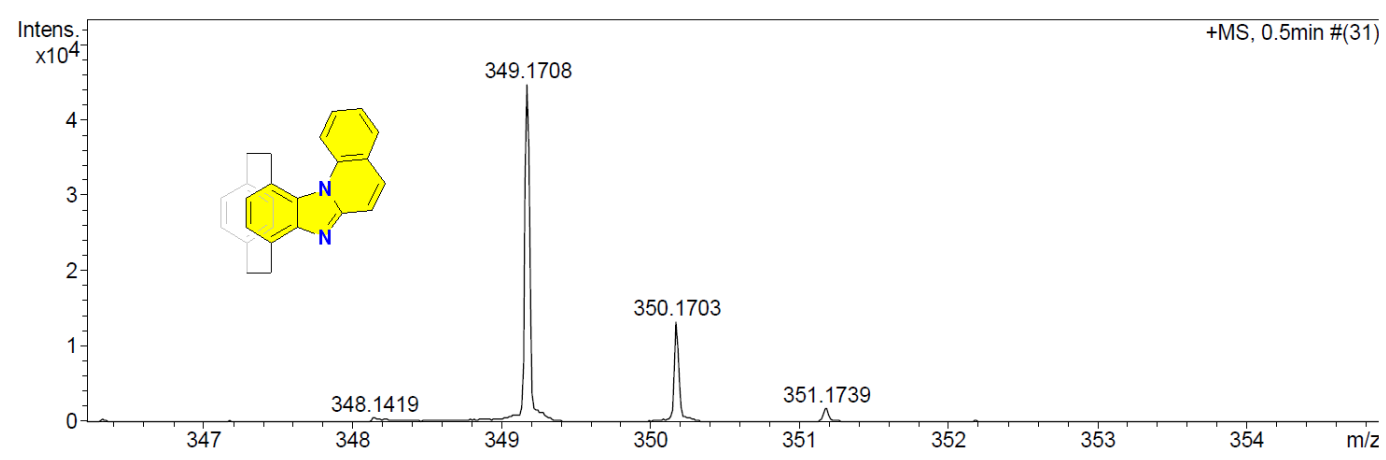


${ }^{1} \mathrm{H}$ NMR of $3 \mathrm{c}\left(500 \mathrm{MHz}, \mathrm{CDCl}_{3}\right)$

员

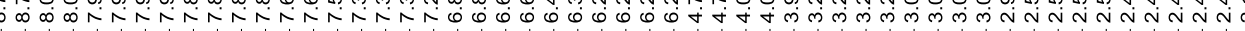

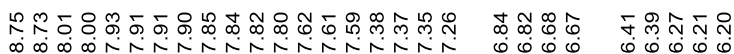

$\underbrace{\infty}$

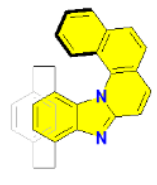

$\left(500 \mathrm{MHz}^{\mathrm{CDCl}} 3\right)$
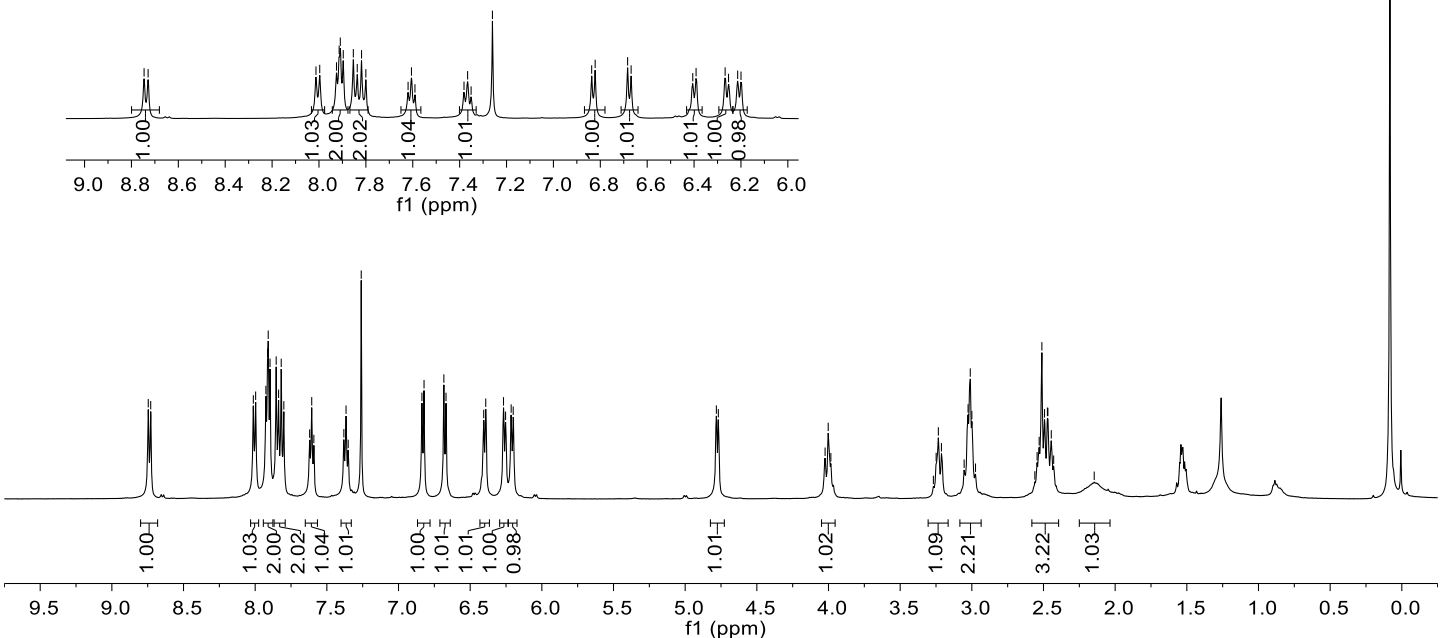

${ }^{13} \mathrm{C}$ NMR of $3 \mathrm{c}\left(126 \mathrm{MHz}, \mathrm{CDCl}_{3}\right)$

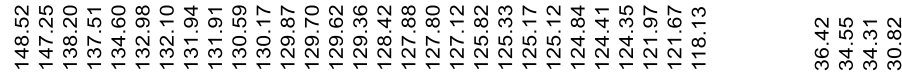

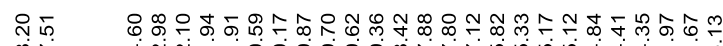

li
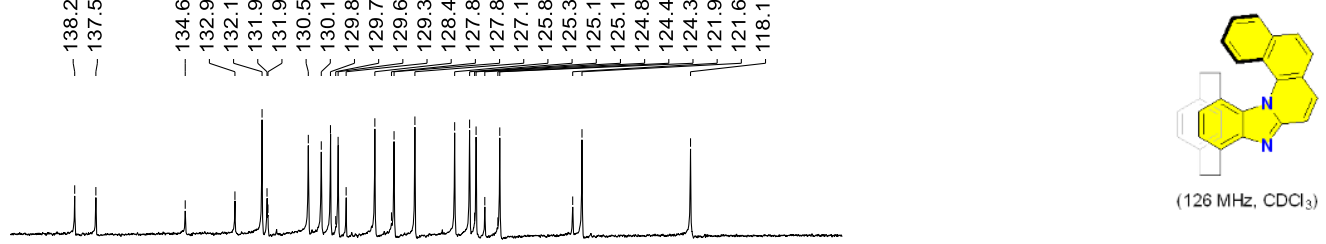

$\left(126 \mathrm{MHz} \mathrm{CDCl}_{3}\right)$

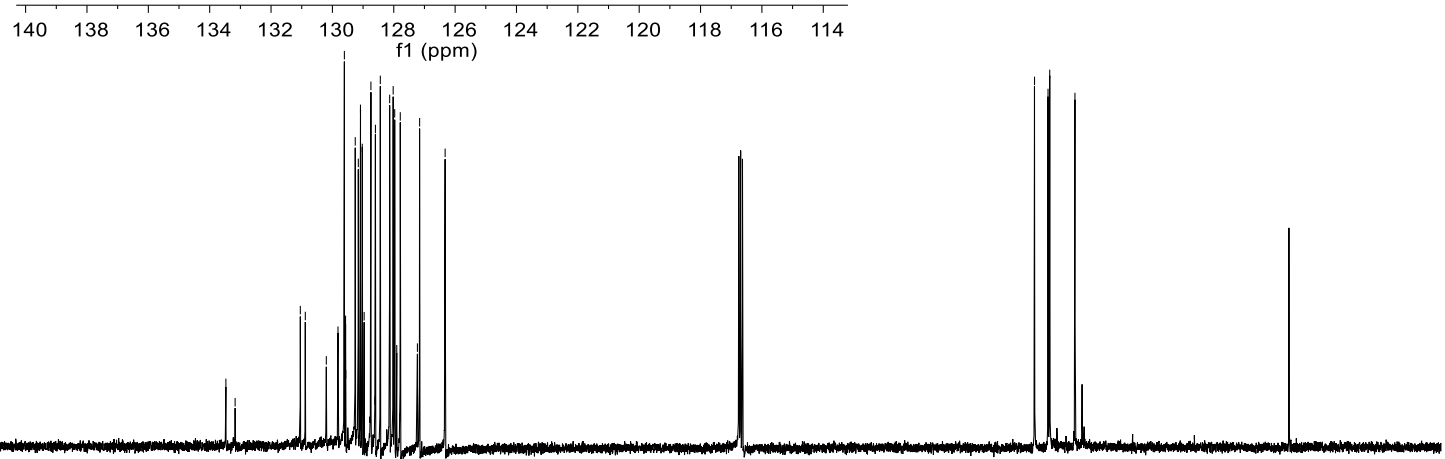

$\begin{array}{lllllllllllllllllllllll}80 & 170 & 160 & 150 & 140 & 130 & 120 & 110 & 100 & 90 & 80 & 70 & 60 & 50 & 40 & 30 & 20 & 10 & 0 & -10 & -1\end{array}$ 


\section{HRMS of 3c}

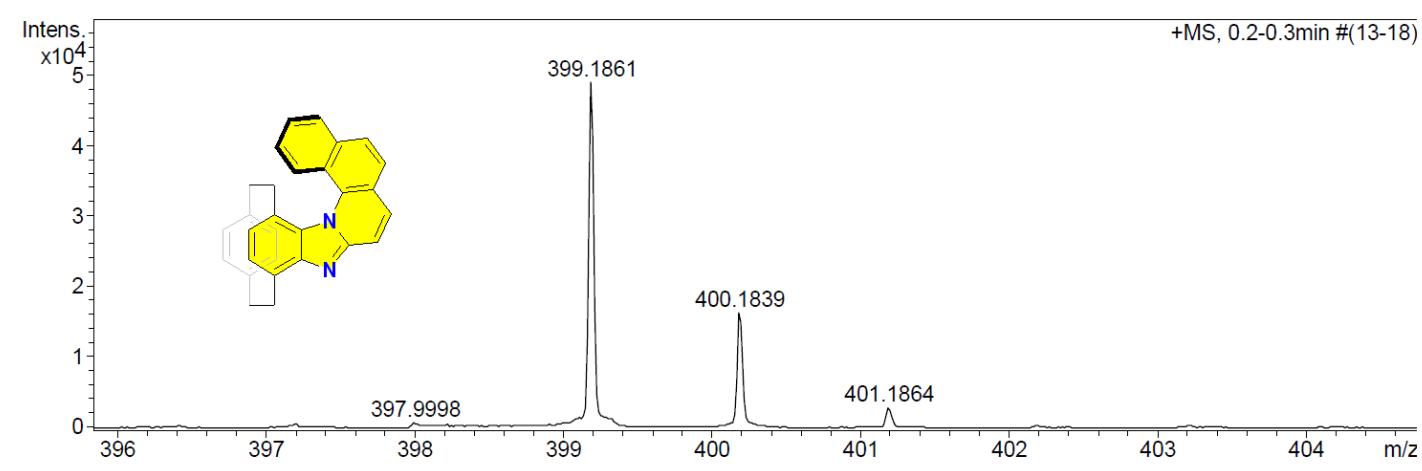

